\title{
ASYMMETRIC DISTRIBUTION OF GAS IN THE LMC AND DYNAMICAL CONDITION FOR GLOBULAR CLUSTER FORMATION
}

\author{
MITSUAKI FUJIMOTO, YASUKI KUMAI \\ Department of Physics \\ Nagoya University \\ Nagoya 464-01 \\ Japan
}

The Large and Small Magellanic Clouds (LMC, SMC) have been in a binary state for the last 10 Gyr, and their centre-of-mass revolves around the Galaxy with a massive dark halo of $10^{12} M_{\mathrm{o}}$. The dashed line in Fig. 1 is a relative orbit of the SMC, approaching the LMC 0.2 Gyr ago with impact parameter of 0.5 to $4 \mathrm{kpc}$ (Murau \& Fujimoto 1980, Fujimoto \& Noguchi 1990). At this close approach, their gas clouds collided hydrodynamically to make shock compressions and coalescences in various mass scale. The ribbon is a computed orbit of a huge gas complex thus generated at $\alpha=5^{h} 40^{m}$ and $\delta=-68^{\circ}$ and reaching the present position.

The 30 Dor is exactly at the apex of the ribbon and the elongated massive CO-complex (Cohen et al. 1988) lies along its axis. Highly asymmetric distributions of CO and HI gases in the LMC seem to be a result of the latest hydrodynamical collision between the LMC and SMC. The 30-Dor region is at a forward end of the huge gas complex in orbital motion, and thus the ram pressure due to the ambient gas would stimulate it to enhance the star formation in its neighbour.

The plane of the ribbon orbit is less tilted by about $20^{\circ}$ against our line of sight, compared with the LMC disk (de Vaucouleurs 1978). Gas clouds, thus coalesced after collision, would move on similar orbits to form a mean disk with the same inclination angle. Many other gas clouds, escaping the hydrodynamical collision with the SMC, would remain nearly in their original disk. When we observe rotation velocities of these two disks of gas, a two-valued rotation curve (Fig. 2) as obtained by McGee and Milton (1966) is reproduced. This curve and, therefore, the global existence of colliding motion of gas clouds with velocity of $\sim 100 \mathrm{~km} \mathrm{~s}^{-1}$ is not accidental but intrinsic to the LMC in strong interaction with the SMC. This is a remarkable difference in dynamical structures of gas between the LMC and the Galaxy.

Unlike the Magellanic Clouds, our Galaxy ceased creating globular clusters 10 Gyr ago. Why the big difference between these galaxies? Fig. 3 (Kumai et al. 1990), shows that LMC globular clusters cover $[\mathrm{FE} / \mathrm{H}]=-2.5$ to 0 , suggesting that the chemical abundance of heavy elements of gas is not related directly to globular cluster formation. Given the difference between the Galaxy and LMC in dynamical gas structure, we present a dynamical condition for globular cluster formation: in any galaxy at any time, if cold gas clouds $>10^{4} M_{\Theta}$ are in a disordered motion of $>100 \mathrm{~km} \mathrm{~s}^{-1}$, globular clusters will be created. The gas layer, compressed by shock collision with relative velocity of $100 \mathrm{~km} \mathrm{~s}^{-1}$, is gravitationally unstable and split into fragments of $10^{5}$ to $10^{6} \mathrm{M}_{\text {e }}$ comparable to the mass of a globular cluster.

In an early phase of the formation of the Galaxy, violent motion must have been present among primordial gas clouds, with random velocities of $>100 \mathrm{~km} \mathrm{~s}^{-1}$, and decayed in some $10^{9} \mathrm{yr}$, because the Galaxy has had no long-lasting nearby disturbers. Globular clusters as observed today 


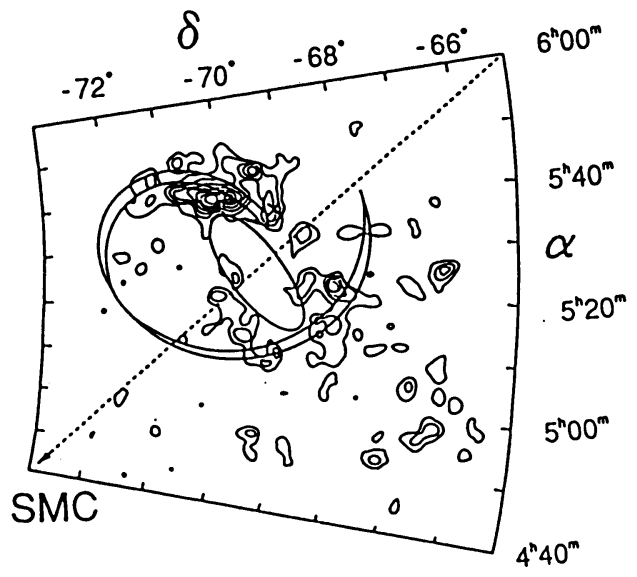

Figure 1. Orbits of the SMC (dashed line) and of a coalesced gas complex (ribbon), superimposed on the optical bar and CO gas complexes (Cohen et al. 1988) of the LMC.

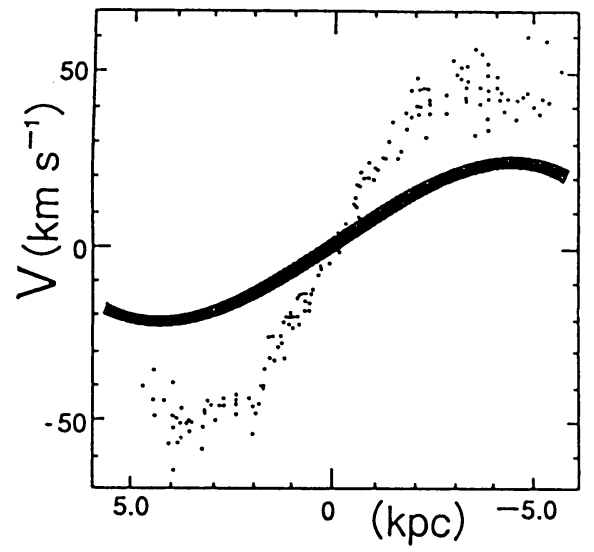

Figure 2. Two-valued rotation curves of gas in the LMC.

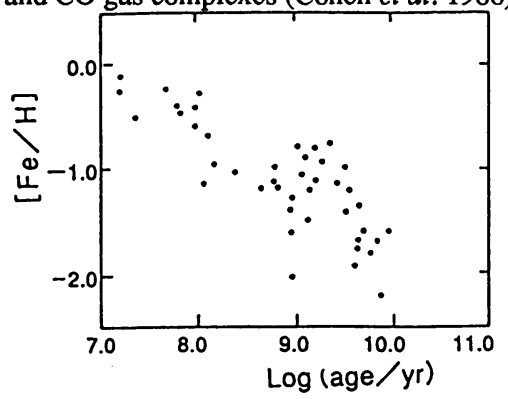

Figure 3. Age-metallicity relation of clusters (Kumai et al. 1990)

with fewer heavy elements, were born only during this early period and their formation ceased quickly with the decay of turbulence.

Young blue globular clusters in M82 would have been generated by the non-circular motion of $>100 \mathrm{~km} \mathrm{~s}^{-1}$ (Cottrel 1977) and resultant shock compression. The massive elliptical M87 is famous for its exceptionally numerous globular clusters (Hanes 1980). It is located at the centre of the Virgo cluster of galaxies, and its huge mass is considered to be due to the merging of a number of galaxies. Virgo cluster galaxies are rich in gas and therefore the galaxies so far attracted to M87 must have also been so. Gaseous clouds tidally torn off from these galaxies in M87 would have made random motion of $>100 \mathrm{~km} \mathrm{~s}^{-1}$ and collided with each other frequently. This dynamical state was a large-scale version of that of the LMC.

\section{References}

Cohen, R.S., Dame, T.M., Garay, G., Montani, J., Rubio, M., Thaddeus, P. (1988), Astrophys. J. 331, L95.

Cottrel, G.A. (1977), M.N.R.A.S. 178, 577.

de Vaucouleurs, G. (1978), Astrophys. J. 131, 265.

Fujimoto, M., Noguchi, M. (1990), Publ. Astr. Soc. Japan in press.

Hanes, D.A. (1980), Globular Clusters, D. Hanes, B. Madore (eds.) (Cambridge UP) p.1.

Kumai, Y., Sabano, Y., Tosa, M. (1990), in prep.

McGee, R.X., Milton, J.A. (1966), Aust. J. Phys. 19, 343.

Murai, T., Fujimoto, M. (1980), Publ. Astron. Soc. Japan 32, 581. 